\title{
Ein kantonales ambulantes Budget - warum nicht?
}

\section{Yves Eggli}

Dr., assoziierter Arzt am IUMSP (Institut universitaire de médecine sociale et préventive / Institut für Sozial- und Präventivmedizin) der Universität Lausanne und niedergelassener Arzt, Mitglied der Expertengruppe "Massnahmen zur Kostendämpfung in der obligatorischen Krankenpflegeversicherung»

Das schweizerische Gesundheitssystem bietet Qualitätsleistungen, aber zu einem hohen Preis (im Jahr 2017 lag dieser bei ca. 10000 Franken/Einwohner). Experten aus der Schweiz und aus Europa gaben jüngst Empfehlungen heraus, darunter u.a. die Einführung einer Höchstgrenze zur Dämpfung des Kostenanstiegs [1]. Die Mehrzahl der Akteure im Gesundheitswesen veröffentlichte daraufhin direkt ein Communiqué gegen ein Globalbudget [2]. Warum diese Eile? Die Sorge der Leistungserbringer um ihre Einkünfte lässt sich zwar nachvollziehen, doch die Vorbehalte der Krankenversicherer der santésuisse verwundert doch sehr. Sind sie nicht besorgt ob dieses Kostenanstiegs im Gesundheitswesen, der möglicherweise von 77 Milliarden Franken im Jahr 2015 auf über 90 Milliarden 2019 ansteigen könnte [3]? Diese Kosten belasten die Haushalte zahlreicher Familien [4] und können in bestimmten Fällen dazu führen, dass die Betroffenen auf die Pflegeleistungen verzichten [5]. Inflationsbereinigt übersteigen die Gesundheitsausgaben der Schweiz pro Einwohner die unserer Nachbarn um 54\% [6]. Die FMH veröffentlichte jüngst Stellungnahmen, in denen vor allem das Risiko von Leistungsbeschränkungen und die Entwicklung einer Zweiklassenmedizin angeprangert werden [7-9].

Obwohl es für den Kostenanstieg im Gesundheitswesen teilweise gute Gründe gibt - vor allem die älter werdende Bevölkerung und der medizinische Fortschritt -, ist er gleichzeitig auch bedingt durch überhöhte Preise (z.B. nicht umgelegte Preissenkungen beim Einkaufspreis bestimmter Ausstattungsobjekte), unnötige Leistungen oder Leistungen ohne entsprechenden Mehrwert [10]. Ein Budgetrahmen im Verbund mit einer Vergütung der jeweils erbrachten Einzelleistung würde die Qualität der Pflege garantieren (Reaktionsfähigkeit der Pflegenden/freie Praxisausübung) und gleichzeitig den Anreiz für eine Vervielfachung der Behandlungen begrenzen. Der Effizienzgewinn könnte daraus erwachsen, dass bei Wahrung des Patienteninteresses eine sinnvolle Nutzung der Leistungen angestrebt und Überversorgung vermieden wird, so wie auf der Smarter Medicine-Liste empfohlen [11].

\section{Wer setzt die Höhe des Budgetrahmens fest und wie geschieht dies?}

Der Budgetrahmen entspricht jenem Betrag, den die Gesellschaft ihm zugesteht. Der oben genannte Expertenbericht empfiehlt (Massnahme 37), Artikel 51 KVG dahingehend zu modifizieren (hervorgehobener Text = neu): «... dass der Kanton als finanzielles Steuerungsinstrument einen Gesamtbetrag für die Finanzierung der Spitäler oder der Pflegeheime oder des ambulanten Sektors festsetzen kann".

Die Schweiz ist ein föderaler Staat, in dem die Kantone über wichtige Vorrechte zur Steuerung der Gesundheitspolitik verfügen. Die Kosten der obligatorischen Krankenpflegeversicherung variieren deutlich von einem Kanton zum anderen, in Extremfällen bis zum doppelten Satz. Grosse Unterschiede gibt es auch bei der Besteuerung.

Die Höhe des Budgetrahmens würde von den kantonalen Behörden unter Berücksichtigung mehrerer Kriterien entschieden. Dazu zählen u.a. der Kostenvergleich zwischen den Kantonen im Laufe der Zeit, Teuerung, Bruttoinlandprodukt, Kaufkraft, demographische Entwicklung, Einbringungen aus dem zu erwartenden medizinischen Fortschritt, Zufriedenheit der Bevölkerung, Wartelisten etc.

\section{Umfang des Budgetrahmens}

Bestimmte ambulante Leistungen wären vom Budgetrahmen auszunehmen, darunter beispielsweise die ambulante Chirurgie, um die Chirurgen nicht dazu $\mathrm{zu}$ veranlassen, die Patienten ins Spital einweisen zu müssen. Diese Frage wäre auch relevant für andere, im Spital zu erbringende Leistungen (Bestrahlungen, Chemotherapie). Ebenfalls ausgenommen werden könnten Dialyse, Physio- und Ergotherapie sowie Ambulanzkosten und Medikamente. So liesse sich vermeiden, dass die Ärzte darauf verzichten, um ihre Einkünfte nicht zu reduzieren. Die häusliche Pflege könnte ebenfalls über einen Budgetrahmen geregelt werden. Allerdings wäre es sinnvoll, diese Regelung 
getrennt von den medizinischen Leistungen vorzunehmen.

\section{Finanzierungsmechanismus}

Der Budgetrahmen wird im Voraus von den zuständigen kantonalen Behörden festgesetzt. Der Taxpunktwert (TARMED, Analysen) berechnet sich, indem der jeweilige Betrag durch die Anzahl der fakturierten Punkte dividiert wird. Gibt es mehr fakturierte Punkte als prognostiziert, wird der für den Kanton geltende Taxpunktwert nach unten revidiert (Tab. 1). Es wird vorläufig ein etwas tieferer Taxpunktwert festgesetzt; der Saldo wird nach Kontoabschluss (Registrierung der letzten Rechnungen) an die Leistungserbringer überwiesen.

Tabelle 1: Beispiel einer Anwendung des Budgetrahmens auf das Rechnungsjahr 2017.

\begin{tabular}{lllll} 
& $\begin{array}{l}\text { CHF } \\
\text { (Mio.) }\end{array}$ & $\begin{array}{l}\text { TARMED- } \\
\text { Punkte } \\
\text { (Mio.) }\end{array}$ & $\begin{array}{l}\text { Tax- } \\
\text { punkt- } \\
\text { wert }\end{array}$ & Jahr \\
\hline 1: Budget-Prognose & 96,0 & 100 & 0,960 & 2016 \\
\hline 2: Vorläufige Rechnung & 93,6 & 104 & 0,900 & 2017 \\
\hline 3: Kontoabschluss & 96,0 & 105 & 0,914 & 2018 \\
\hline
\end{tabular}

In diesem Zusammenhang ist es wichtig, dass Ärzte die erforderlichen Kontrollinstrumente zur Hand haben, um ihre Tätigkeit zu regulieren. Die Daten zur Fakturierung würden zentral gesammelt, um den jeweiligen Stand zu analysieren. Dabei könnte das Augenmerk vor allem auf die Reduzierung unnötiger Leistungen, überhöhte Abrechnungen, die Einführung eines Dialogs mit den teuersten Ärzten (je nach Schwere der Erkrankung der Patienten) und die Einkommensentwicklung pro Fachgebiet und Region gerichtet werden. Die Analyse der medizinischen Behandlungsmethoden würde ebenfalls eine Anpassung der in den Qualitätszirkeln zu diskutierenden Themenkomplexe und der Orientierung der Weiterbildung bedingen.

Bei sinkendem Behandlungsvolumen könnte beispielsweise jenen Ärzten ein zusätzliches Entgelt vergütet werden, die am meisten zur Kostenkontrolle bei den Medikamenten beigetragen haben (durchschnittlich günstigere Verschreibungen für ein und dieselbe Therapiekategorie).

\section{Führen Globalbudgets zu einer effizien- teren Kostenkontrolle?}

Kantone, die bei der Finanzierung ihrer Spitäler oder ihrer Alten- und Pflegeheime einen Budgetrahmen einsetzen, konnten ihre Kosten in diesen Bereichen kontrollieren, gelegentlich auch mit Erhöhungen, die jedoch gewollt und demokratisch abgestimmt waren. Einige bezweifeln die Wirksamkeit von Globalbudgets, wie sie beispielsweise in Deutschland oder in den Niederlanden eingeführt wurden [2]. In diesem Zusammenhang kommt es auf die Voraussetzungen an, unter denen eine Umsetzung des jeweiligen Budgetrahmens effizient wäre:

- automatische Taxpunktwertänderung bei Veränderungen im Leistungsumfang;

- der Zielbereich ist sorgfältig abzugrenzen, um gegenläufige (sprich nach oben gerichtete) Anreize zu vermeiden;

- die Leistungserbringer müssen in Bezug auf die Verteilung der zugewiesenen Beträge verantwortlich handeln lernen (starke Einflussnahme auf die angenommenen Regeln, keine zu grosse Tarifgemeinschaft, damit sich die Ärzte kennen, Datentransparenz).

\section{Führt dies zu verlängerten Wartezeiten, einer Rationierung der Pflege und zu einer Zweiklassenmedizin?}

Der Einsatz eines Budgetrahmens führt nicht zwingend zu einer Rationierung. Vertretbare Budgeterhöhungen sind möglich, und der sparsame Umgang mit den verfügbaren Ressourcen ermöglicht die Finanzierung von innovativen Leistungen mit hohem Mehrwert.

Der Expertenbericht [1] macht deutlich, dass das Hauptproblem in der Vervielfachung von Behandlungen mit geringem Mehrwert zu suchen ist. Wer könnte dies am besten vermeiden? Der Patient verfügt häufig nicht über ausreichendes Wissen, Versicherer und Staat sind in der Regel nicht genau genug informiert, um darüber zu entscheiden. Da bleiben letztlich noch die Ärzte, die am besten entscheiden können, was empfohlen werden kann und was nicht, und die auch einen kritischen Blick auf die medizinischen Behandlungsmethoden werfen können.

Das schweizerische Gesundheitssystem ermöglicht gegenwärtig eine individualisierte Pflege und einen allgemeinen Zugang zu den Leistungen. Ein übertriebener Kostenanstieg könnte drastische Massnahmen zur Kostenkontrolle nach sich ziehen, die dem Patienten und dem Arzt weniger Wahlfreiheit lassen. Die richtigen Massnahmen zur Vermeidung von Exzessen heute tragen zum Erhalt der Behandlungsfreiheit von morgen bei.

\section{Besteht das Risiko, dass die Kränksten zurückbleiben?}

Es besteht kein Grund zur Annahme, dass ein Budgetrahmen die Prioritäten des Pflegesystems modifizie- 
ren würde. Ganz im Gegenteil, er würde Korrekturmassnahmen ermöglichen, sollten Probleme mit dem Zugang zur Pflege auftreten. So ist heute beispielsweise ein gewisses Desinteresse seitens der Ärzte zu verzeichnen, wenn es um komplexere Patienten geht, die z.B. Hausbesuche oder Besuche am Abend oder am Wochenende betreffen. Dies schadet dem Pflegesystem und führt gelegentlich zu vermeidbaren Hospitalisierungen. Finanzanreize würden die gerechte Vergütung jener Ärzte ermöglichen, die diese Verfügbarkeit garantieren, gegebenenfalls im Verbund mit Kollegen, die sich regelmässig über den Gesundheitszustand ihrer kränksten Patienten informieren. Die Liste dieser Ärzte könnte veröffentlicht werden, damit die Patienten ihre Wahl treffen können.

Solche Regelungen sind sensibel zu handhaben und wären innerhalb der Ärzteschaft zu diskutieren, so dass sich alle darin wiederfinden. Und auch die Patientenverbände und die Kunden könnten sich einbringen und Optimierungen vorschlagen.

\section{Führt dies zu einer Verstaatlichung der Medizin?}

Die Einführung eines kantonalen ambulanten Budgetrahmens ändert nichts am Gesundheitssystem. Nur die Regeln zur Festsetzung der Taxpunktwerte sind davon betroffen. Ein Budgetrahmen im Verbund mit einer Vergütung der jeweils erbrachten Einzelleistung würde die reagible Herangehensweise in Bezug auf die Anforderungen der Patienten gewährleisten und gleichzeitig den Anreiz für eine Vervielfachung unnötiger Behandlungen vermeiden. Ein solcher Rahmen ist nicht politisch orientiert (links oder rechts), sondern unterstellt die Frage der Gesundheitsausgaben den demokratischen Kräften.

\section{Werden die Patienten nicht mehr behan- delt, wenn die Ausgaben den Rahmen übersteigen?}

Diese Sorge, die in Deutschland bei der Einführung des Globalbudgets zum Ausdruck gebracht wurde, ist nicht relevant. Kein dem Budgetrahmen unterstelltes Spital und kein Alten- oder Pflegeheim hat zum Jahresende den Betrieb eingestellt, weil es sich in einer schwierigen Finanzlage befand. Der für die Einzelbehandlung vergütete Arzt hat immer den Vorteil, die Leistung erbringen zu können, sonst würde er sein Einkommen schmälern. Im Übrigen geht es - entgegen gewisser Pressemeldungen - nicht darum, Budgetrahmen pro Arzt oder Behandlung einzuführen. Es geht ausschliesslich um ein Globalbudget.

\section{Fazit}

Die Einführung eines kantonalen ambulanten Budgetrahmens würde eine verbesserte Kontrolle des Kostenwachstums ermöglichen und dabei gleichzeitig die Freiheit der Behandlung des Arztes gewährleisten. Auf eine Regulierung des Behandlungsumfangs basierte Lösungen führen zu einem Verlust an Freiheit. Ausschliesslich tarifbasierte Massnahmen sind in den letzten Jahren an ihre Grenzen gestossen.

\section{Anmerkung}

Die in diesem Artikel zum Ausdruck gebrachten Ansichten und Meinungen verpflichten nur den Autor.

\section{Literatur}

1 Vom Bundesrat in Auftrag gegebener Expertenbericht. Massnahmen zur Kostendämpfung in der obligatorischen Krankenpflege versicherung. Bern: BAG, 2017.

2 http://www.santesuisse.ch/de/details/content/globalbudgets sind_leichtfertige_experimente_zu_lasten_der_patientinnen und patienten 1349/

3 www.tdg.ch/suisse/sante-cap-10-000-fr-habitant-franchi/ story/15624963

4 www.24heures.ch/sante/sante/Les-primes-maladies-asphyxientle-budget-d-une-famille-sur-deux/story/16680807

5 www.tdg.ch/suisse/Un-habitant-sur-quatre-renonce-a-se-soigner-/story/25875369

6 Strupler P. Kosten des Gesundheitssystems steigen stärker als in den Nachbarländern. Die Volkswirtschaft. 23.2.2017. http:// dievolkswirtschaft.ch/de/2017/02/strupler-03-2017fr/

7 Schlup J. Wie die Politik das Rationieren an den Arzt delegieren möchte. SÄZ. 2017;98:47.

8 Schulte U. Globalbudget im Gesundheitswesen - Ein Problem als Lösung. SÄZ. 2017;98:25.

9 Kesseli B. Globalbudgets - eine Scheinlösung? SÄZ. 2017;98:47.

10 Gerber M, Kraft E, Bosshard C. Overuse - unnötige Behandlungen als Qualitätsproblem. SÄZ. 2016;97:236-43.

11 Capaul R. Garantie d'une médecine abordable et de grande qualité. Quel est le lien entre qualité et smarter medicine? Primary and Hospital Care. 2017;17:3 S. 